\title{
Design and Implementation of Simulation System for Safety Accident Case Based on Immersive Virtual Reality Technology
}

\section{De-Gang ZHANG ${ }^{1, a}$, Ze-He LI ${ }^{1}$, Yan-Wei XIAO², Long JIANG ${ }^{1}$, Da-Da WANG ${ }^{1}$ and Guo-Ping WANG ${ }^{3, b,{ }^{*}}$}

\author{
${ }^{1}$ Education Training Evaluation Center of Yunnan Power Grid Co., Ltd, Beijing, China \\ 2 State Grid Zhejiang Electric Power Corporation, Zhejiang, China \\ ${ }^{3}$ Beijing Kedong Electric Control System Co. Ltd., Beijing, China \\ aemail: zdg.csg@hotmail.com, bemail: 13681220069@163.com \\ ${ }^{*}$ Corresponding author
}

\begin{abstract}
Keywords: Electric power safety training; safety accident case; substation safety case tool; immersive stereoscopic display; virtual human interaction.
\end{abstract}

\begin{abstract}
In view of the problems existing in the safety education and training of Substation, this paper designs a simulation training system for electric power safety accidents. This paper introduces the hardware and software scheme of the system, focusing on production safety accident case, the system uses the immersive 3D display, virtual human motion capture and interactive substation multi-persons' collaborative technology based on field application shows that the system through immersive visual experience and natural interaction mode to realize safety education and training efficient, so that students in a virtual training environment personally on the scene, the profound experience of the serious consequences of violation of safety rules of operation, so as to strengthen the ability to operate, regulate the production and operation.
\end{abstract}

\section{Introduction}

With the rapid development of China's power grid, substation as an important part of the power grid, the security awareness and technical quality of the substation operation and maintenance personnel play an important role in the safe and stable operation of the substation. The theory of separation and practical operation of substation operation safety production training, learning theory, abstract, monotonous and boring, inconvenient for students to understand, in the practical operation of the process, students of operation rules and points of unfamiliar, easy to misuse, cause the rising rate of accident phenomenon, the traditional training mode has been unable to meet the needs of electric power safety training. The difference between the training and the actual operation of the environment, the effect of poor safety training, resulting in the process of practical operation, the students of the operating procedures and points unfamiliar, easy to misuse [1]. As a result, it is necessary for the electric power safety training to be highly interactive, situational and experiential.

Immersive virtual reality simulation technology based on virtual reality interactive devices, such as stereo projection, helmet display and motion capture equipment, virtual environment to build a highly immersive and interactive nature of participants. In recent years, with the maturity and the consumption level of virtual reality hardware immersive virtual reality technology has introduced [2], interactive devices such as Oculus Rift, HTC VIVE virtual reality helmet virtual reality helmet and Microsoft Kinect somatosensory interactive devices [3]. It is a new research hotspot and development trend to build a highly immersive substation simulation training system based on Immersive Virtual Reality Technology.

Safety accident case simulation training system is designed in this paper, using the current substation simulation training and immersive virtual reality of the latest technological achievements, the use of three-dimensional projection, virtual reality helmet, position tracking, motion capture and 
other virtual reality interactive devices, to achieve a highly immersive and interactive training methods for electric power safety simulation training.

\section{Overall System Design}

\section{1 Software System}

Safety case production tools, power grid simulation, substation simulation, 3D projection system, immersive virtual interactive system, training management of software system, time control and information interaction, the software module of the simulation logic synchronization through simulation platform to realize the bottom. Figure 1 shows the system software architecture.

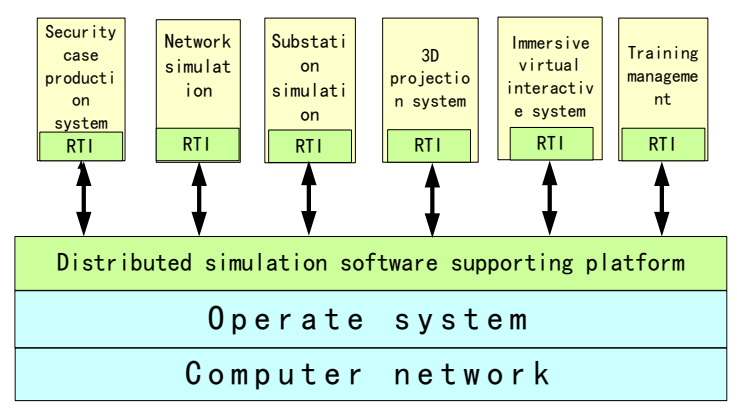

. Fig. 1 Structure diagram of immersive simulation training system

1) The safety case making tools provide a visual tool to make safety education case for teachers, teachers through the power system in the safety production of casualty accidents, typical production accident analysis, summed up the operation errors appear in the course of operation, there is a risk, the use of safety accident case making tools to visual interactive way of editing making correct specification for training operation and wrong operation compared to the double simulated case, let students understand different consequences of different operations, improve their training effect.

2) Grid simulation through flow calculation, according to the simulation cycle must calculate the power grid equipment telemetry data and signaling data and sent through the network to the substation simulation module, telemetry data network equipment, including the current value and voltage value, active power and reactive power data, power telesignal data including circuit breaker and isolation switch state. At the same time, the power network simulation module receives the control information of the circuit breaker and the isolating switch [4], which is sent by the substation simulation module through the network communication, and updates the state of the grid model.

3)Substation simulation based on mechanism model of substation equipment [5], using advanced 3D engine development, realize the three-dimensional modeling of the substation electrical equipment, auxiliary equipment, environmental scene and virtual human, running situation and realistic simulation of substation equipment and one or two substation station monitoring and control system.

4)The three-dimensional projection through $3 \mathrm{D}$ image mosaic $3 \mathrm{D}$ image conversion program for conversion of substation scene images are output to the multi-channel stereo projector stereoscopic display three-dimensional image is formed by stereo projector stereo arc screen.

5) The spatial position information of immersive virtual interactive module to obtain precise real-time motion capture captures participants limb key points, limb including knee and elbow, waist and neck top position, and limb joint position information is mapped to the virtual human space, by calculating the corresponding joint people into virtual space rotation, spatial operation of virtual human, then through gesture recognition accurate collision 3D virtual human hand computing equipment and substation detection and virtual human, accurately complete the operation behavior of 
the students, such as press the reset button, twisting the handle, ultimately converted into operation in Substation.

6)The function of the training management module includes the training plan, the training process control and management, and the evaluation of the results.

\subsection{Hardware System}

The hardware of this system includes stereo projection, stereo arc screen, virtual reality helmet, motion capture, simulation workstation and network switch. The specific hardware structure is shown in Figure 2 below.

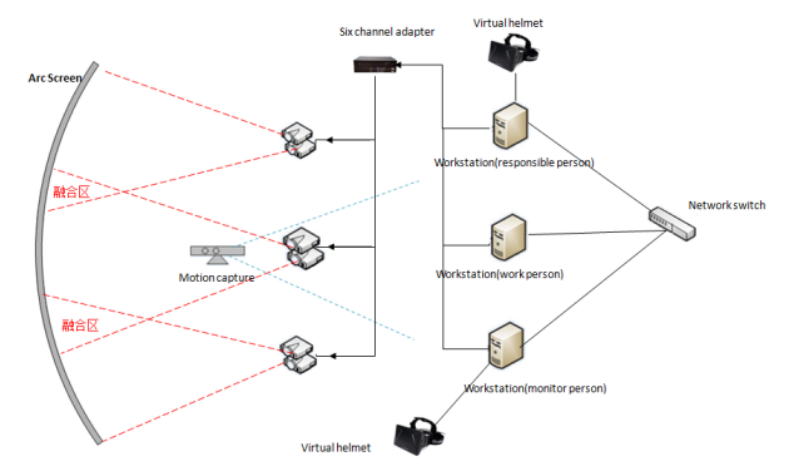

Figure 2 Structure diagram of hardware system

1)System configuration 3 Simulation workstation. The main workstation is used for the operator to carry out the virtual operation, and the video signal is transmitted to the fusion machine and output through the 6 projection to the large arc screen, so as to synthesize the $3 \mathrm{D}$ image. The main workstation is connected with the motion capture device through the data line, which can capture the human motion capture data in real time and drive the virtual human and the virtual scene device to realize the interactive operation. Workstation 2 is connected to the virtual helmet or screen for the guardian to interact with the virtual scene. Workstation 3 is connected to a virtual helmet or screen for the user to interact with the virtual scene.

2)System configuration 3 stereo projector, respectively through a video line connection and receiving hardware image 3 component image and projected onto the screen on the screen stereo fusion arc output, students can by wearing 3D glasses, immersive experience one or two substation equipment normal, abnormal and accident conditions and operation process of substation equipment virtual inspection, case processing, inspection, operation and accident process of roaming.

3) The space coordinates and motion motion capture data acquiring position key user motion in real time, and real-time data will be transferred to the simulation machine to update the virtual characters in the corresponding joint rotation angle, so as to realize the control of virtual characters and scene interaction.

4) The position tracker virtual reality helmet matching, through the real-time tracking of position sensing points on the helmet, spatial position and orientation and transmit real-time access to real-time updates of helmet three-dimensional image simulation workstation helmet display.

5) Data exchange in distributed system based on network switch

\section{Implementation of Key Technologies}

\section{1 Safety Accident Case Making Technology}

In this paper, the virtual reality technology is used in the case of the security of the substation, the user can use the model to build the typical safety production case. A typical case is a collection of a series of key steps and key events in the process of operation, this project uses a typical case of said objects to the data flow chart, the case said steps and primitive event case primitives directly connected by 
directed arcs to represent a case primitive time sequence. The case flow chart uses the expandable XML format to store and read data.

\section{2 Immersive Stereoscopic Display Technology}

This project uses two kinds of immersive stereoscopic display technology of virtual reality helmet and three-dimensional arc screen projection, which can not only carry out personal immersion training for the training staff, but also open the training process.

Virtual reality helmet built-in two small display screen respectively for stereoscopic display around the eyes, a wide range of closed people's vision system through the three-dimensional image with display is decomposed into two images with parallax of the left eye, and are output to the virtual reality helmet in the left eye to achieve binocular stereo immersive display screen. The software of the system is achieved through the establishment of two virtual camera view corresponding to left eye in $3 \mathrm{D}$ scene, the 3D scene image of the left and right eye corresponding to the virtual camera synchronization output to a virtual reality helmet eye screen to achieve binocular stereo imaging.

Stereo arc screen display required output resolution image resolution than single projector, so it is necessary to 3D image mosaic projection through multiple projectors, this system has developed the 3D image mosaic conversion program, the program of 3D graphics rendering of the input image, and the image is converted into a single 3D 3D for multi-channel projection output the three-dimensional image display according to the projection parameters, then the multidimensional images are output to the different projectors and projection to the stereo arc scene, resulting in three-dimensional output effect.

\section{3 Virtual Human Interaction Technology Based on Motion Capture}

Human motion data of virtual human motion capture interactions including simulation system of motion by optical motion capture system for training personnel based on real-time data recording and then mapping movement into the virtual human in the virtual scene, to drive the virtual human in the virtual scene and make consistent training of personnel movement. The interactive technology is not simply to confirm or return to this selective operation, but to reflect the movement of their own staff.

The original motion data, motion capture system to get the motion data with redundant information, in order to drive the motion of virtual human, this project first data denoising and eliminate redundant motion data from motion, and a large number of data extract can correctly reflect the motion data of virtual human motion process, and then deal with the data were processed according to the mapping of the virtual human model, and ultimately drive the virtual human motion data.

\section{4 Multi Person Cooperation Technology in Substation Operation}

The substation operation process including the guardian, the operator, the person in charge, the system support people at the same time respectively to the guardian, the operator and the person in charge of the role of interaction, in full accordance with the actual operation of the substation work flow, together to complete a task. The system realizes the task mode of multi person cooperation. In this mode, a number of trainees can log into the training scene with different roles and work together in a team to complete the task of a multi participant. In the process of collaborative work, the students in accordance with their respective roles and tasks in the simulation system on the simulation of practical operation, users use text, voice and other communication methods for communication.

The system developed by the communication function of centralized client / server mode to realize multi-user collaboration, in the use of multi person cooperative function, all client first need to establish a reliable network links with collaborative server module, in the multi-persons'collaborative function during the operation, the occurrence of any client on the interactive operation requires the interactive operation the transmission of information through the network to the server, the server receives a postprocessing operation information and operating information forwarded to other clients to be cooperative, processing and forwarding server, the client is responsible for sending and receiving operation information synchronization information and update the scene. 


\section{Practical System Application}

The results of this research has been successfully applied to the Yunnan Power Grid Co education evaluation center, has conducted simulation training safety accident case of production related personnel, training personnel through the immersion of the system to provide interactive experience, can be a profound experience of risk points in the task of substation operation and maintenance as well as the accident phenomenon does not comply with safety regulations brought, so as to enhance the students safety accident case training impression.

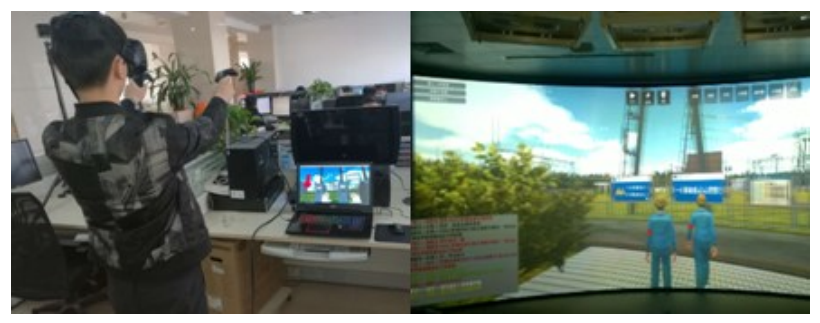

Fig. 3 System application effect diagram

\section{Summary}

Safety accident case simulation training system for immersive virtual reality technology using immersive virtual reality model to new re interpretation of power substation safety production based on education, through the establishment of substation one or two scene model, and the case and restore the true reproduction of the scene, interact with safe operation and training through a virtual reality helmet, motion capture system and 3D stereo projection system and Virtual Substation Scene Simulation, without affecting the normal operation of the power network, so that employees can experience the actual production accident, risk factors exist in the working environment of all kinds of sense of touch and do not regulate the operation of the consequences, to solve the deficiency of the traditional sense of the scene and the lack of user training mode experience, so as to improve the training effect.

\section{References}

[1] Li Yuqing, Wu Huizhong, Lin Chang. Research and implementation of [J]. system simulation in virtual environment of substation simulation and training system of the 2006, 18 (S1):123-126. .

[2] Hou Jun, Li Yuqing, Lin Chang substation years. Research on key technology of 3D interactive scene simulation [J]. power system technology, 2005, 29 (9):70-75.

[3] Huang Xiantun, Yan Jin. The problems and Countermeasures of the training methods of typical accident cases in the safety training of electrician special operations personnel [J]. science and technology information, 2008,201-203.

[4] Yang Xuanhuai, Wang Y Y, Wang Guoping, et al. Power system simulation platform based on high level architecture [J]. power system technology, 2009, 33 (11):98-103.

[5] Hai Xiaotao, Lin Wei, Gao Feng, et al. Integrated simulation training system for Northwest 750 $\mathrm{kV}$ AC / DC hybrid power grid [J]. power system technology, 2012. 